\title{
Epigenetic modulation of the MAPK pathway prevents isoflurane-induced neuronal apoptosis and cognitive decline in aged rats
}

\author{
LEI HUANG, HAI-BIN FANG, HUI-HUI CHENG, SHENG-LAN MEI, YUN-PING CHENG, \\ YAO LV, QING-TAO MENG and ZHONG-YUAN XIA
}

Department of Anesthesiology, Renmin Hospital of Wuhan University, Wuhan, Hubei 430060, P.R. China

Received August 19, 2019; Accepted April 17, 2020

DOI: $10.3892 / \mathrm{etm} .2020 .9162$

\begin{abstract}
Isoflurane is a broadly used inhalation anesthetic that causes cognitive impairment in rodent models as well as humans. Although previous studies suggested an association between isoflurane exposure and neuro-inflammation, apoptosis and mitochondrial dysfunction, the pathogenesis of isoflurane-induced cognitive decline remains elusive. In the present study, 22-month-old male Sprague-Dawley male rats $(n=96)$ were divided into three groups: Control (Cont), isoflurane (ISO) and MS-275 pre-treated groups. The rats were sacrificed following exposure to isoflurane and a cognitive test. The hippocampus of each animal was harvested for quantitative PCR, TUNEL staining and western blot analysis. Histone deacetylases (HDAC)-1, -2 and -3 exhibited a significant increase at the gene and protein expression levels, whereas negligible mRNA expressions were observed for genes HDAC 4-11 ( $\mathrm{P}>0.05$; compared with Cont). Pre-treatment with the HDAC inhibitor MS-275 significantly inhibited the increase in TUNEL-positive cells induced by isoflurane exposure (70.72\% decrease; $\mathrm{P}<0.001$; compared with ISO). Furthermore, MS-275 significantly decreased caspase-3 and Bax expression levels while increasing Bcl-2 protein expression. The isoflurane-induced changes in the MAPK pathway signaling proteins ERK1/2, JNK and p38 were also reversed with MS-275 pre-treatment. Finally, in a Morris water maze test, the time to find a hidden platform was reduced in MS-275 pre-treated rats, compared with the ISO group. Therefore, the present study provided insight into the effect of isoflurane exposure on neuronal apoptosis pathways, as well as cognitive decline via epigenetic programming of MAPK signaling in aged rats.
\end{abstract}

Correspondence to: Dr Zhong-Yuan Xia, Department of Anesthesiology, Renmin Hospital of Wuhan University, 238 Jiefang Road, Wuhan, Hubei 430060, P.R. China

E-mail: roxannebrowningyjz@yahoo.com

Key words: isoflurane, MAPK, epigenetics, histone-deacetylase, cognitive, apoptosis, hippocampus

\section{Introduction}

Prolonged isoflurane exposure has been implicated in postoperative cognitive dysfunction, particularly in the elderly $(1,2)$. Several previous studies have demonstrated the effect of isoflurane exposure on neurotoxicity and cognitive impairment due to a rise in neuronal inflammation and apoptosis, especially in the hippocampus region of the brain (2-5). However, the molecular mechanisms that lead to isoflurane-induced cognitive decline in aged rats remain elusive.

Epigenetic modification regulates the transcription of genes that are essential for the maintenance of memory processes (6). Accumulating evidence supports the crucial role of chromatin-mediated epigenetic regulation of behavior and memory formation $(7,8)$. The most common mechanism of epigenetic modification involves histone acetylation, which is altered dynamically during the course of learning and memory formation (9). Histone acetylation is endogenously achieved by maintaining a balance between the activity of histone acetyltransferases and histone deacetylases (HDACs). An increase in histone acetylation results in a more relaxed chromatin structure and enhances the transcription of genes vital for long-term memory, whereas histone deacetylation induces a negative effect (10). It has also been found that epigenetic variations inside the brain are crucial for short- and long-term behavioral adaptation to a wide range of environmental stimuli (11). These external environmental stimuli can stimulate intracellular pathways that directly remodel the epigenome and lead to differences in gene expression and neuronal function in the early development as well as the adult stages of life $(12,13)$. Isoflurane exposure is one such external stimulus, and thus, targeting histone acetylation using HDAC inhibitors (HDACis) before isoflurane exposure might reverse neurotoxicity and cognitive decline. One such HDACi is MS-275, which is specific to HDACs 1-3. In contrast to other HDACi, MS-275 can enter the brain even at low doses, and raise the levels of acetylated histone 3 (Ac-H3). In addition, MS-275 has a long half-life (14).

Epigenetic programming is largely reliant on the activation of transcription factors binding to promoter regions of downstream target genes, and on intracellular signaling cascades, such as the MAPK pathway. The MAPK family of 
serine-threonine protein kinases comprises ERK, p38 and JNK as its main members (15). The dynamic expression of MAPK signaling proteins in the mature nervous system, mainly in the hippocampus, suggests a role for the MAPK cascade in synaptic plasticity, learning and memory formation (16-18). Previous studies have also provided insight into the function of MAPK pathways in reducing sevoflurane-induced neurotoxicity $(19,20)$. However, to the best of the authors' knowledge, no study to date has investigated how epigenetic modification of MAPK signaling pathways might lead to functional changes in hippocampal neurons following isoflurane exposure.

Therefore, in the present study, it was hypothesized that pre-treatment with an HDACi could alleviate isoflurane-induced neuronal apoptosis and cognitive decline in aged rats. The present study identified a direct association between epigenetic programming and MAPK signaling following isoflurane exposure.

\section{Materials and methods}

Animals. Male, 22-month-old Sprague-Dawley rats (280-300 g; $\mathrm{n}=96$ ) were purchased from The Shanghai SLAC Laboratory Animal Co., Ltd. All the experiments in the present study were approved by the local ethical committee of Renmin Hospital of Wuhan University and performed according to the guidelines presented in the Declaration of the National Institutes of Health Guide for Care and Use of Laboratory Animals. All animals were kept under a $12 \mathrm{~h}$ light/dark cycle with a relative humidity of $40-70 \%$ in a temperature-regulated room at $22-24^{\circ} \mathrm{C}$ with unrestricted access to food and water.

Isoflurane exposure. The animals were exposed to isoflurane as previously described (21). Briefly, the animals were placed in a chamber pre-filled with $3 \%$ isoflurane in $30 \% \mathrm{O}_{2}$ for the first $10 \mathrm{~min}$ followed by $1.5 \%$ isoflurane exposure to maintain constant anesthesia for $2 \mathrm{~h}$ on 7 consecutive days. The concentrations of gases inhaled and exhaled were regularly monitored using the Datex Capnomac Ultima stand-alone multi gas analyzer (GE Healthcare).

MS-275 administration and experimental design. The animals were divided into three groups: Control (Cont), isoflurane (ISO) and MS-275-treated (n=32 in each group). For hippocampal-dependent spatial memory evaluation, rats $(n=8$ in each group) were randomly chosen for Morris water maze (MWM) training before test exposures. Prior to ISO exposure, MS-275 (2 mg/kg; Sigma-Aldrich; Merck KGaA) and PBS were administered intraperitoneally to the animals in the MS-275 and ISO groups, respectively. The rats in the Cont group were not exposed to isoflurane but were kept in the chamber filled with $30 \% \mathrm{O}_{2}$ for $2 \mathrm{~h}$ on 7 consecutive days. All rats then recovered in a chamber with $30 \% \mathrm{O}_{2}$ at $37^{\circ} \mathrm{C}$ for $20 \mathrm{~min}$. The rats were sacrificed following isoflurane exposure and cognitive testing by $\mathrm{CO}_{2}$ inhalation based on the AVMA Guidelines on Euthanasia (22). The hippocampus of each animal was harvested, rapidly frozen in liquid nitrogen and stored at $-80^{\circ} \mathrm{C}$ for further analysis.

Reverse transcription-quantitative PCR (RT-qPCR). For RT-qPCR analysis, total RNA was extracted from the hippocampus using the RNA-spin ${ }^{\mathrm{TM}}$ Total RNA Extraction kit (Intron Biotechnology, Inc.). RNA concentrations were measured using the NanoDrop 1000 system (Thermo Fisher Scientific, Inc.). cDNA synthesis was carried out using the First Strand cDNA Synthesis kit (Thermo Fisher Scientific, Inc.) with the following cycling conditions: Digestion at $37^{\circ} \mathrm{C}$ for $2 \mathrm{~min}$, annealing at $65^{\circ} \mathrm{C}$ for $5 \mathrm{~min}$, RT at $50^{\circ} \mathrm{C}$ for $10 \mathrm{~min}$, and enzyme inactivation $85^{\circ} \mathrm{C}$ for $5 \mathrm{~min}$. Subsequently, RT-qPCR was performed on the ABI 7500 Real-time PCR system (Applied Biosystems; Thermo Fisher Scientific, Inc.) using SYBR Green PCR Master Mix (Thermo Fisher Scientific, Inc.). The thermocycling conditions for RT-qPCR were: $95^{\circ} \mathrm{C}$ initial template denaturation, 40 cycles of $95^{\circ} \mathrm{C}$ denaturation and $60^{\circ} \mathrm{C}$ annealing/extension. GAPDH was used as the endogenous control to normalize mean $\mathrm{Cq}$ values using the $2^{-\Delta \Delta \mathrm{Cq}}$ method (23). The sequences of the primers used are presented in Table SI.

TUNEL assay. Hippocampi from each rat were fixed with $4 \%$ paraformaldehyde for $48 \mathrm{~h}$ at $4^{\circ} \mathrm{C}$, paraffin-embedded, then sectioned at a thickness of $5 \mu \mathrm{m}$. The Dead End ${ }^{\mathrm{TM}}$ fluorometric TUNEL system kit (Promega Corporation) was used to perform the assay according to the manufacturer's guidelines. Hoechst dye (Hoechst 33342; Thermo Fisher Scientific, Inc.) was used as a nuclear stain and diluted to $1 \mu \mathrm{g} / \mathrm{ml}$ in PBS from $10 \mathrm{mg} / \mathrm{ml}$ stock prepared in deionized water. Samples were stained for $10 \mathrm{~min}$ at room temperature, with protection from light. SlowFade ${ }^{\mathrm{TM}}$ Glass Soft-set Antifade Mountant (Thermo Fisher Scientific, Inc.) was used for mounting. TUNEL-positive cells were examined using NIS-Elements BR imaging processing and analysis software version 4.00 (Nikon Corporation), and 10 microscopic fields were analyzed per group (magnification, x200). TUNEL-positive cell density in the CA1 region of the brain was evaluated by dividing the number of TUNEL-positive cells by the area of that region of the brain.

Western blotting. Protein for western blot analysis was isolated from the hippocampal tissue samples homogenized in ice-cold RIPA Pierce ${ }^{\text {TM }}$ buffer (Thermo Fisher Scientific, Inc.) supplemented with protease inhibitor at final $1 \mathrm{X}$ concentration (Halt ${ }^{\mathrm{TM}}$ Phosphatase Inhibitor Cocktail, Thermo Fisher Scientific, Inc.). Protein concentrations were measured using a BCA protein assay kit (Thermo Fisher Scientific, Inc.). Proteins were then separated by SDS-PAGE on $10 \%$ gels and $20 \mu \mathrm{g}$ total protein/lane was transferred to a nitrocellulose membrane. After the transfer, the blot was kept in 5\% w/v non-fat dry milk blocking solution for $1 \mathrm{~h}$ at room temperature, then incubated with primary antibodies overnight at $4^{\circ} \mathrm{C}$. The primary antibodies used were: Anti-HDAC1 (1:400; Abcam; cat. no. ab53091), anti-HDAC2 (1:400; Abcam; cat.no.ab32117), anti-HDAC3 (1:400; Abcam; cat.no.ab32369), anti-cleaved caspase-3 (1:1,000; Abcam; cat. no. ab2302), anti-Bcl-2 (1:1,000; Abcam; cat. no. ab32124) anti-Bax (1:1,000; Abcam; cat. no. ab32503), anti-phospho-ERK1/2 (p-ERK1/2; 1:1,000; Abcam, cat. no. ab223500), anti-ERK1/2 (1:1,000; Abcam; cat. no. ab17942), anti-phospho-JNK (p-JNK;1:1,000; Abcam; cat. no. ab47337), anti-JNK (1:1,000; Abcam; cat. no. ab199380), anti-phospho-p38 (p-p38;1:1,000; Abcam; cat. no. ab4822) and anti-p38 (1:1,000; Abcam; 

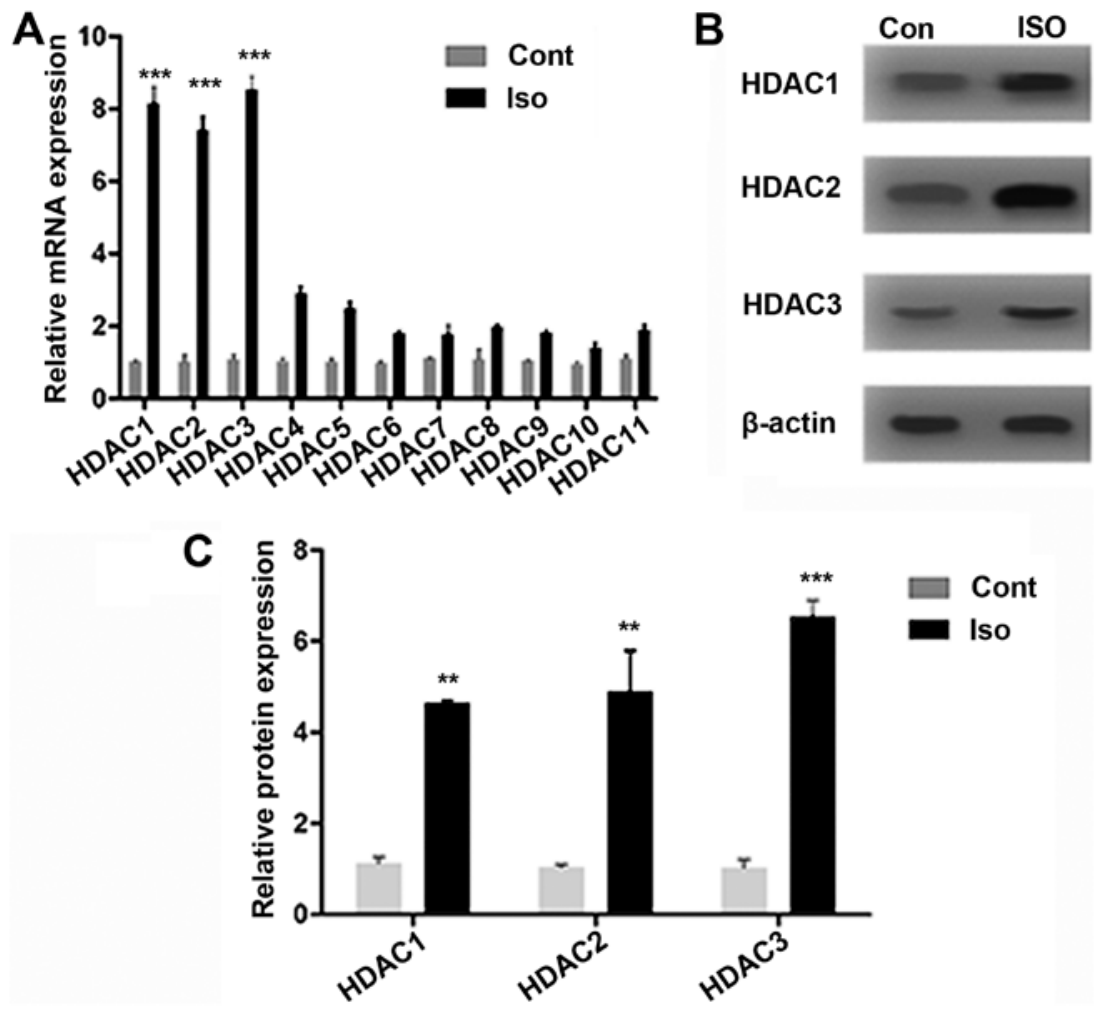

Figure 1. Quantification of HDAC mRNA expression. (A) mRNA expression of HDAC1-11, where HDACs 1-3 levels increase in comparison with other HDACs. (B) HDAC1-3 protein expression using western blot analysis. (C) Quantification of HDAC1-3 band intensities confirming their upregulation following isoflurane exposure. Data are presented as the mean \pm SEM. ${ }^{* *} \mathrm{P}<0.001$ and ${ }^{* * * *} \mathrm{P}<0.001$ vs. respective Cont. Cont, control group; ISO, isoflurane group; HDAC, histone deacetylase

cat. no. ab31828). Anti- $\beta$-actin (1:500; Abcam; cat. no. ab1801) was used as the internal control for each sample. After incubation with primary antibodies, the blot was washed three times with PBS $+0.1 \%$ Tween-20 and incubated with horseradish peroxidase-conjugated goat anti-mouse IgG secondary antibody (1:500; Abcam; cat. no. ab97023) at room temperature for $2 \mathrm{~h}$. Protein bands were detected using an enhanced chemiluminescent kit (cat. no. EK1001; CliniSciences, Nanterre) and the band density was visualized and quantified using Image J software version 1.48 (National Institutes of Health). The quantification is presented as the ratios between protein band densities of the protein of interest with respect to the internal control protein band density. The protein expression of p-ERK1/2, p-JNK or p-p38 was normalized to total ERK1/2, JNK or p38, respectively. The signal intensity of bands of other proteins of interest were normalized to $\beta$-actin.

MWM experiments. The MWM test was performed to evaluate spatial learning and memory in rodents (24). A circular pool with a diameter of 180 - and 50-cm deep was filled with opaque water at a temperature of $26^{\circ} \mathrm{C}$. A round platform with a diameter of $10 \mathrm{~cm}$ was immersed $2 \mathrm{~cm}$ beneath the water surface in one quadrant. The position of the platform was unaltered for each rat. In total, 8 rats from each group were randomly chosen for MWM training. Rats were trained on 4 trials/day for 5 consecutive days before receiving anesthesia. The minimum interval between every trial was $15 \mathrm{~min}$. If the rats failed to find the hidden platform within $90 \mathrm{sec}$, they were manually guided for $30 \mathrm{sec}$ towards the hidden platform. After training, the rats were subjected to the experimental protocol as described. On the 7th day of isoflurane exposure, the platform was raised $2 \mathrm{~cm}$ above the surface of water so that the platform was visible to rats. This also helped to evaluate the effect of isoflurane exposure and MS-275 pre-treatment on non-spatial factors like visual acuity, sensorimotor performance and impetus on cognitive function. Each trial was considered complete either when the rat successfully climbed to the platform or when the trail duration reached $90 \mathrm{sec}$. After spending $30 \mathrm{sec}$ on the platform in between trials, mice were put in a cage warmed with electric heating pad for $5 \mathrm{~min}$ to allow recovery. The time taken for each trial in a day was averaged and statistically analyzed.

Statistical analysis. The data are presented as the mean \pm SEM. One-way ANOVA was used for comparisons between variables, followed by Bonferroni post hoc test using GraphPad Prism 5.0 (GraphPad Software, Inc.). $\mathrm{P}<0.01$ and $\mathrm{P}<0.001$ were used to indicate a statistically significant difference. Each experiment was performed in triplicate and repeated thrice.

\section{Results}

HDAC expression following isoflurane exposure. The expressions of different HDAC isoforms were quantified following isoflurane exposure. RT-qPCR was performed for all HDAC isoforms (HDAC1-11), identifying strong signals for HDAC1 (8.15-fold; $\mathrm{P}<0.001)$, HDAC2 (7.4-fold; $\mathrm{P}<0.001)$ and HDAC3 $(8.55$-fold; $\mathrm{P}<0.001)$ genes, compared with Cont. The increase in mRNA expression was notably lower forHDAC4 (1.87-fold; P>0.01) and for HDACs 5-11 (Fig. 1A). HDAC1-3 

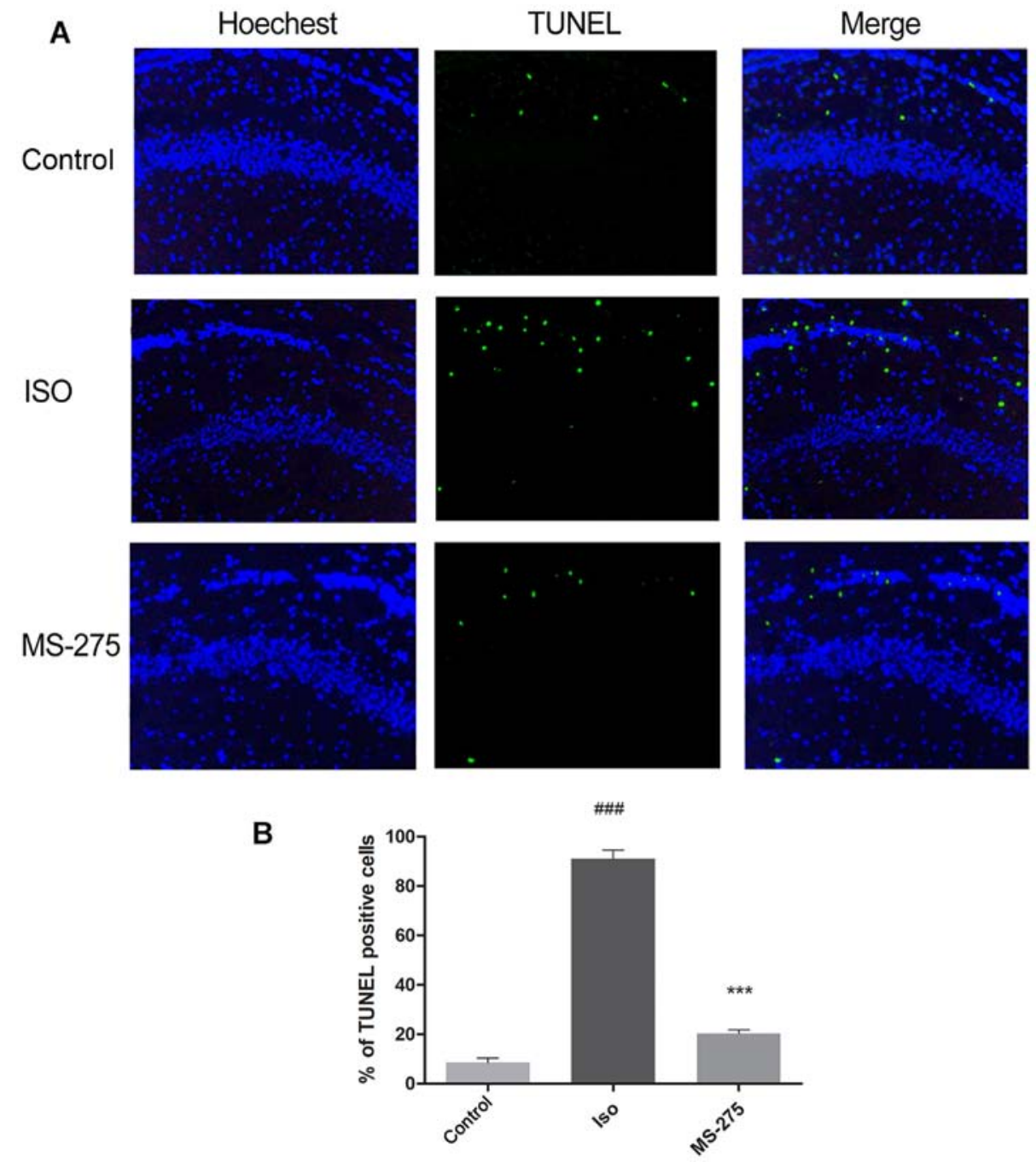

Figure 2. MS-275 inhibited neuronal apoptosis in rats exposed to isoflurane. (A) Representative fluorescent images of the CA1 region of hippocampus, where blue stain indicates Hoechst and green stain indicates TUNEL-positive cells. Magnification, x200. (B) Quantification of the number of TUNEL-positive cells in Cont, ISO and MS-275 pre-treated isoflurane-exposed rats. Data are presented as the mean \pm SEM. ${ }^{\# \# *} \mathrm{P}<0.001$ vs. Cont; ${ }^{* * * *} \mathrm{P}<0.001 \mathrm{vs.} \mathrm{ISO}$. TUNEL, terminal deoxynucleotidyl-transferase-mediated dUTP nick end labeling; Cont, control group; ISO, isoflurane group.

protein expression significantly increased, compared with Cont (Figs. 1B and $\mathrm{C} ; \mathrm{P}<0.001$ and $\mathrm{P}<0.01$ ), suggesting isoflurane-induced epigenetic dysregulation. Notably, HDAC8, which is also a class-I HDAC, did not exhibit a significant change in mRNA expression after isoflurane exposure when compared with the Cont group. This could be attributed to the fact that HDAC8 is not recruited to chromatin through large multi-protein complexes like HDAC1-3 (25). The localization of class-I HDACs in the nucleus (26) has allowed for the elucidation of how HDAC inhibition modulates MAPK signaling proteins in isoflurane-induced aged rats.

MS-275 pre-treatment decreases isoflurane-induced neuronal apoptosis in the hippocampus. To determine the effect of MS-275 on isoflurane-induced neuronal apoptosis, TUNEL staining was performed on the CA1 region of the hippocampus. Following isoflurane exposure, the number of TUNEL-positive cells increased by $82.47 \%$ in the hippocampal CA1 region compared with Cont (Fig. 2; P<0.001). Pre-treatment with MS-275 significantly inhibited the increase in TUNEL-positive cells after isoflurane exposure.
A70.72\% decrease in TUNEL-positive cells was observed in rats pre-treated with MS-275, compared with the ISO group (Fig. 2; $\mathrm{P}<0.001)$. These results demonstrated that MS-275 could alleviate isoflurane-induced neuronal apoptosis.

MS-275 pre-treatment alters the expression of apoptotic regulatory proteins. The effect of isoflurane exposure and MS-275 pre-treatment on neuronal apoptosis was further assessed by evaluating the expression levels of proteins directly involved in apoptosis. Protein expression of cleaved caspase-3, Bcl-2 and Bax in the whole hippocampus was quantified using western blot analysis (Fig. 3A). Isoflurane significantly increased the levels of cleaved caspase-3 (7.1-fold; P<0.001; Fig. 3B) and Bax (3.98-fold increase; $\mathrm{P}<0.001$; Fig. 3D), compared with Cont. In addition, isoflurane also reduced $\mathrm{Bcl}-2$ protein levels, relative to Cont (0.795-fold, $\mathrm{P}<0.01$; Fig. 3C). However, pre-treatment with MS-275 significantly decreased caspase-3 (5.975-fold; $\mathrm{P}<0.001$; Fig. 3B) and Bax protein levels (3.18-fold; $\mathrm{P}<0.001$; Fig. 3C), compared with ISO. Bcl-2 protein expression was also increased following MS-275 treatment, compared with ISO (1.145-fold; P<0.001; Fig. 3C). 


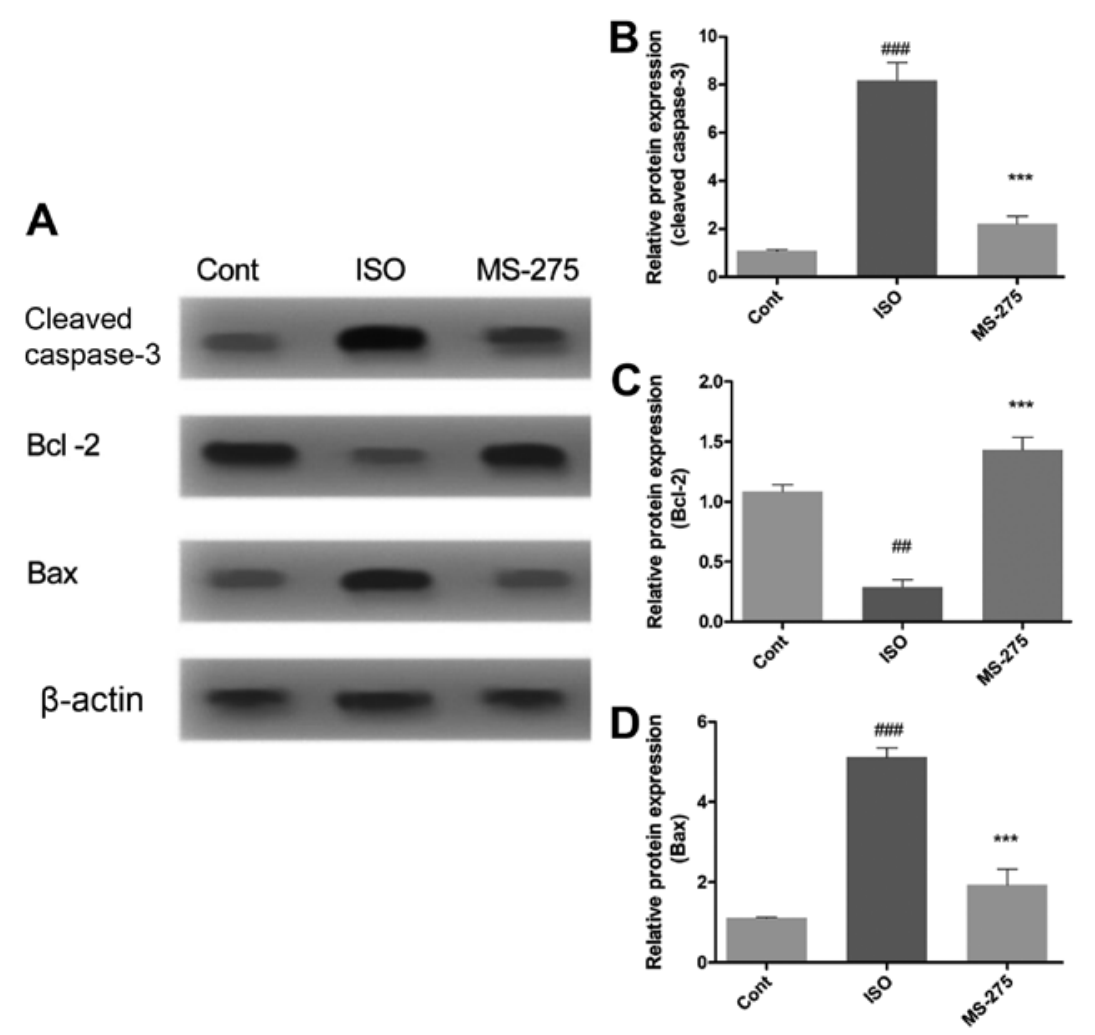

Figure 3. Expression of apoptosis regulatory proteins, cleaved caspase-3, Bcl-2 and Bax. (A) Representative density bands after western blotting of cleaved caspase-3, Bcl-2 and Bax proteins was performed. (B) Quantification of cleaved caspase-3 relative expression. (C) Quantification of Bcl-2 relative expression. (D) Quantification of Bax relative expression. Data are presented as the mean $\pm \mathrm{SEM}$. ${ }^{\# \#} \mathrm{P}<0.01$ and ${ }^{\# \# \#} \mathrm{P}<0.001$ vs. Cont; ${ }^{* * *} \mathrm{P}<0.001$ vs. ISO. Cont, control group; ISO, isoflurane group.

MS-275 pre-treatment reverses isoflurane-induced variations in MAPK signaling protein levels. Isoflurane significantly reduced protein expression of p-ERK1/2 $(\mathrm{P}<0.01)$, while increasing the expression of $\mathrm{p}-\mathrm{JNK}(\mathrm{P}<0.001)$ and $\mathrm{p}-\mathrm{p} 38$ $(\mathrm{P}<0.001)$, compared with Cont (Fig. 4). However, pre-treatment with HDACi MS-275 reversed the isoflurane-induced changes in MAPK signaling proteins. Indeed, p-ERK1/2 expression was increased, compared with the ISO group (Fig. 4B). Furthermore, MS-275 reduced the isoflurane-induced increase of p-JNK proteins (Fig. 4C) and p-p38 proteins (Fig. 4D). These results suggested that MS-275 directly influenced the expression of MAPK signaling proteins following isoflurane exposure.

MS-275 pre-treatment improves isoflurane-induced spatial memory impairment. The MWM test is a highly sensitive test which evaluates cognitive function, especially hippocampus-dependent learning and memory in rodents (24). In the present study, aged rats in each group could find the hidden platform prior to isoflurane exposure following MWM training for 5 days. In comparison with the latency on day 1, a significant decrease in latency was detected on day 3 in all rats $(\mathrm{P}<0.001)$. On day 5 of training, the animals in all three groups were able to find the hidden platform within $30 \mathrm{sec}$ (Fig. 5A).

After the training phase, the rats either received ISO or did not (serving as the Cont group). A third group was treated with MS-275 prior to isoflurane exposure. On day 6 of isoflurane exposure, the ISO group took a longer time (close to the maximum time of $120 \mathrm{sec}$ permitted for each animal) to find the hidden platform, compared with the Cont group (113.4 sec; $\mathrm{P}<0.001$ vs. $27.1 \mathrm{sec}$ in the Cont group; Fig. 5B). However, MS-275 treatment significantly reduced the amount of time required to find the platform submerged under the water surface compared with the ISO group $(46.4 \mathrm{sec} ; \mathrm{P}<0.001)$. When the platform was raised on day 7 such that it was visible to the rats, the latency to find the platform relatively reduced in all groups. Nonetheless, the MS-275 pre-treated group exhibited a significant cognitive recovery, compared with the ISO group (23.5 and $91.3 \mathrm{sec}$, respectively; $\mathrm{P}<0.001)$. This suggested that MS-275 pre-treatment had a substantial role in alleviating cognitive decline after isoflurane exposure.

\section{Discussion}

Isoflurane is a broadly used inhalation anesthetic that has been reported to cause cognitive impairment in rodent models as well as humans (1-5). Although these previous studies suggested an association between isoflurane exposure and neuro-inflammation, apoptosis and mitochondrial dysfunction, the pathogenesis of isoflurane-induced cognitive decline needs extensive investigation. Therefore, in the present study, the effect of isoflurane exposure on neuronal apoptosis as well as cognitive decline via epigenetic modulation of MAPK signaling was investigated in aged rats.

Epigenetic modification regulates the transcription of genes that are essential for the maintenance of memory processes (6). Histone acetylation, one of the most studied forms of epigenetic modification, is achieved by maintaining 
A

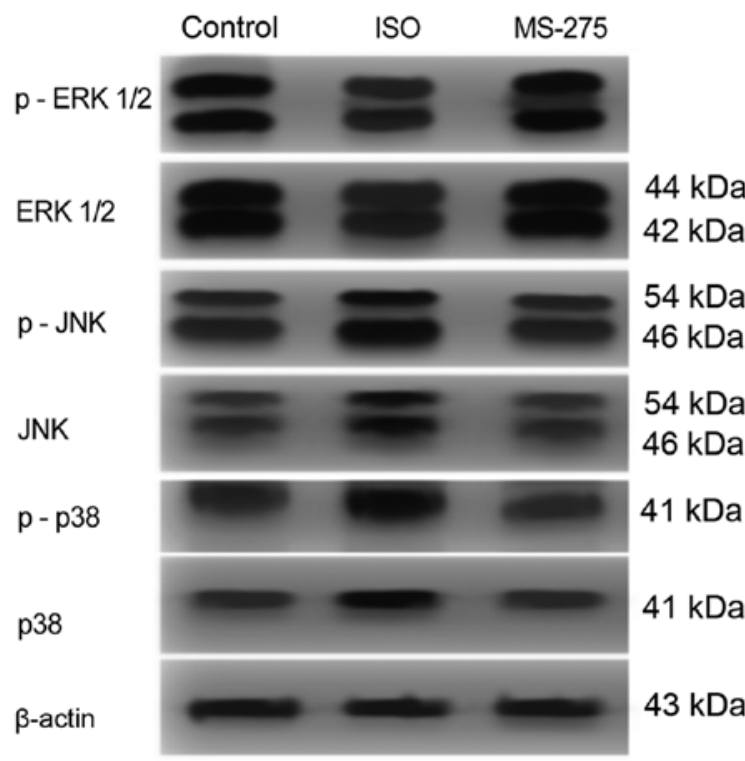

B

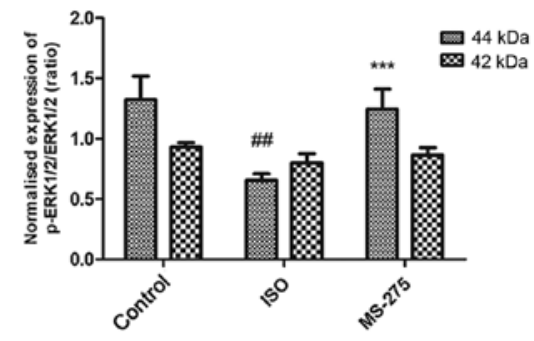

C

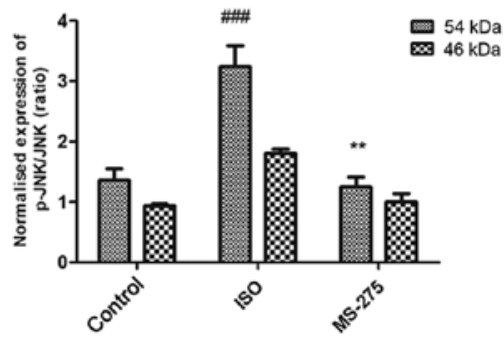

D

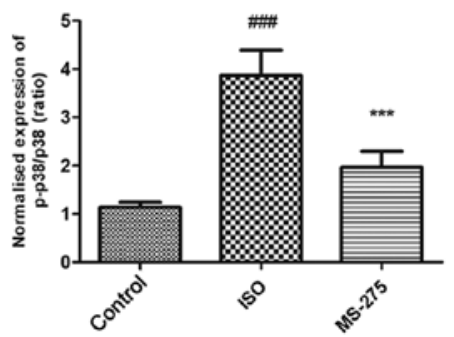

Figure 4. Effect of MS-275 treatment on expression of MAPK signaling proteins following isoflurane exposure. (A) Western blot analysis of MAPK signaling proteins p-ERK1/2/ERK1/2, p-JNK/JNK and p-p38/p38. (B-D) Quantification of the band intensities by normalizing the expression values of the ratios of the following proteins: (B) p-ERK1/2/ERK1/2, where each bar represents the ratio of the normalized expression values between p-ERK1/2 and ERK1/2 of isoforms 44 and $42 \mathrm{kDa}$; (C) p-JNK/JNK, where each bar represents the ratio of the normalized expression values between p-JNK and JNK of isoforms 54 and $46 \mathrm{kDa}$; (D) Ratio of normalized expression values between p-p38 and p38 (41 kDa). Data are normalized and presented as the mean \pm SEM. ${ }^{\# \#} \mathrm{P}<0.01$,

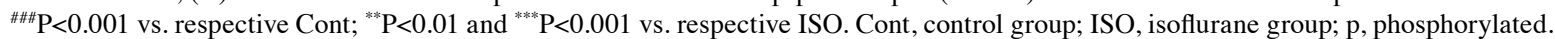
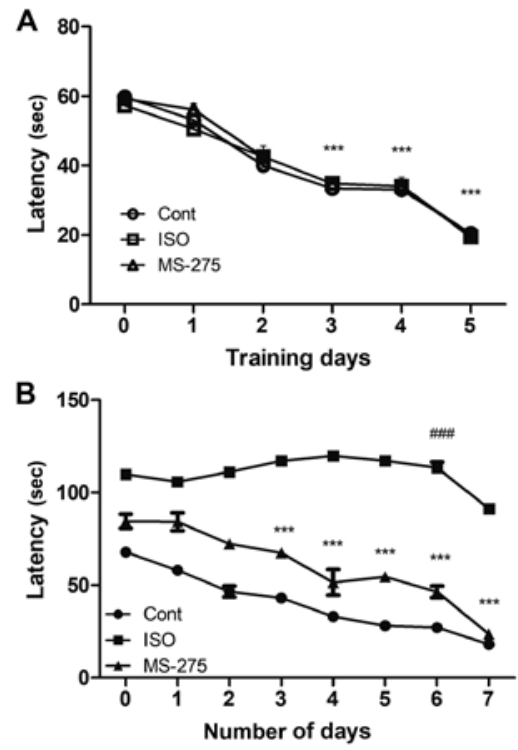

Figure 5. Effect of isoflurane exposure on cognitive impairment in aged rats. A Morris water maze test was carried out and the time taken for the mice to locate the platform hidden/visible on the water surface was recorded. (A) Latency in seconds for the training of rats for 5 days before isoflurane exposure. (B) Latency in seconds to locate the platform after isoflurane exposure. The MS-275 pre-treated group took significantly less time to locate the platform. Data are presented as the mean $\pm \mathrm{SEM}$. ${ }^{\# \# \#} \mathrm{P}<0.001$ vs. Cont; ${ }^{* * *} \mathrm{P}<0.001$ vs. ISO. Cont, control group; ISO, isoflurane group. a balance between histone acetyltransferases and HDACs. Previous studies suggested that the deregulation of histone acetylation in the hippocampus leads to cognitive decline via the suppression of primary genes involved in learning and memory (7-10).

In the present study, isoflurane exposure caused epigenetic dysregulation with the subsequent disruption of neuronal as well as cognitive function. In congruence with the hypothesis of the present study, aged rats exposed to isoflurane exhibited upregulation of class-I HDACs (HDAC1, -2, and -3), while HDAC4-11 showed low expression in the ISO group. Notably, HDAC8 did not exhibit significant change in the expression following isoflurane exposure, unlike the other class-I HDACs. This could be due to the fact that, unlike HDACs 1-3, HDAC8 is not recruited to chromatin through large multi-protein complexes and is completely active as a standalone class-I HDAC (25). Thus, the results of the present study suggested that isoflurane exposure increased histone deacetylation, specifically via HDAC1-3, which may result in a compromised epigenetic milieu.

Moreover, the present study also demonstrated the restoration of histone acetylation using the HDACi MS-275, which is specific to HDAC1-3. In contrast to other HDACi, MS-275 has a long half-life and can enter the brain even at low doses, raising Ac-H3 levels in the brain (14). A recent study demonstrated 
that MS-275 improved autism-induced synaptic and social discrepancies through the epigenetic modulation of associated genes, such as surface NMDARs and actin regulators (27). Thus, MS-275 pre-treatment was used to inhibit HDAC1-3 and study its effect on MAPK signaling proteins, neuronal apoptosis and cognitive function following isoflurane exposure.

Isoflurane predominantly affects the hippocampal region of the brain and induces neurotoxicity, leading to severe hippocampal lesion and an abnormal response to contextual fear conditioning (28). In the present study, MS-275 pre-treatment alleviated isoflurane-induced neuro-apoptosis in the hippocampus of the aged rats. Furthermore, MS-275 pre-treatment reduced isoflurane-induced levels of cleaved caspase-3 (total caspase was not measured) and Bax protein, while increasing Bcl-2 expression. A MWM test also demonstrated that MS-275 pre-treatment prevented isoflurane-induced cognitive and spatial memory impairments. A previous study demonstrated the efficiency of MS-275 in enhancing memory and altering depression-mediated behavior (29); thus, the present study further supported the role of MS-275 in isoflurane-induced cognitive impairment in aged rats. However, cognitive decline was only evaluated by the MWM test, a well-established behavioral test to evaluate spatial learning and memory in rodents. Therefore, the present study would benefit from future studies connecting neuronal architecture, synapse number and neurotransmitters with other cognitive tests.

One of the critical objectives of the present study was to investigate the association between the inhibition of $\mathrm{HDACl}$, -2 , and -3 with MAPK signaling protein expression following isoflurane exposure. MS-275 altered the expression of MAPK signalingproteinsp38, JNK, and ERK1/2, which may have accounted for neuroprotection and cognitive recovery in aged rats exposed to isoflurane. MS-275 suppressed p38 expression in E-11 cells, which demonstrated its efficacy against rheumatoid arthritis (30). Another study demonstrated the importance of highly selective class-I HDACi in the suppression of nuclear ERK1/2 signaling (31). These studies highlight the potential action of MS-275 on MAPK signaling proteins.

The JNK signaling pathway is implicated in neuronal apoptosis triggered by several brain injury stimuli, such as ischemia/reperfusion and ethanol $(32,33)$. Activated JNK phosphorylates c-Jun (a nuclear substrate) and also modulates the transcription of apoptosis-related genes, including $\mathrm{Bcl}-2$ family members that form part of the non-nuclear JNK pathway (32). JNK activation has been demonstrated to be involved in isoflurane-induced neuronal apoptosis (34). The present study suggested that MS-275 pre-treatment deactivated the JNK nuclear pathway by preventing isoflurane-induced increase of phosphorylation of JNK. Additionally, MS-275 also inhibited the JNK non-nuclear pathway by preventing the isoflurane-induced increase of Bax and downregulation of Bcl-2 expression, thereby reversing the isoflurane-induced increase of caspase-3. These results demonstrated the critical role of MAPK signaling in the neuroprotection of MS-275 against the effects of isoflurane.

In summary, the present study demonstrated that pre-treatment with the HDACi MS-275 alleviated isoflurane-induced neuronal apoptosis and cognitive decline in aged rats. The present study may also provide insight for future research involving direct association of epigenetic programming and other neuro-inflammatory pathways, such as nuclear factor- $x \mathrm{~B}$ signaling following isoflurane exposure in aging animals.

\section{Acknowledgements}

Not applicable.

\section{Funding}

The present study was supported by The National Natural Science Foundation of China (grant no. 81671948) and The Guidance Fund of Renmin Hospital of Wuhan University (grant no. RMYD2018M18).

\section{Availability of data and materials}

All data generated or analyzed during this study are included in this published article.

\section{Authors' contributions}

LH, HBF, HHC and ZYX conceptualized and designed the experiments. LH, HBF, HHC and SLM performed the experiments. YPC, YL and QTM performed the statistical analysis and provided technical assistance for the study. ZYX revised the manuscript critically and approved the final version for submission. All authors read and approved the final manuscript.

\section{Ethics approval and consent to participate}

All the experiments in the present study were approved by the local ethical committee of Renmin Hospital of Wuhan University and performed according to the guidelines presented in the Declaration of the National Institutes of Health Guide for Care and Use of Laboratory Animals.

\section{Patient consent for publication}

Not applicable.

\section{Competing interests}

The authors declare that they have no competing interests.

\section{References}

1. Zhang B, Tian M, Zhen Y, Yue Y, Sherman J, Zheng H, Li S, Tanzi RE, Marcantonio ER and Xie Z: The effects of isoflurane and desflurane on cognitive function in humans. Anesth Analg 114: 410-415, 2012.

2. Kong F, Chen S, Cheng Y, Ma L, Lu H, Zhang H and Hu W: Minocycline attenuates cognitive impairment induced by isoflurane anesthesia in aged rats. PLoS One 8: e61385, 2013.

3. Lin D and Zuo Z: Isoflurane induces hippocampal cell injury and cognitive impairments in adult rats. Neuropharmacology 61: 1354-1359, 2011

4. Cao Y, Li Z, Ma L, Ni C, Li L, Yang N, Shi C and Guo X: Isoflurane-induced postoperative cognitive dysfunction is mediated by hypoxia-inducible factor- $1 \alpha$-dependent neuroinflammation in aged rats. Mol Med Rep 17: 7730-7736, 2018.

5. Zhang S, Hu X, Guan W, Luan L, Li B, Tang Q and Fan H: Isoflurane anesthesia promotes cognitive impairment by inducing expression of $\beta$-amyloid protein-related factors in the hippocampus of aged rats. PLoS One 12: e0175654, 2017. 
6. Zhang TY and Meaney MJ: Epigenetics and the environmental regulation of the genome and its function. Annu Rev Psychol 61: 439-466, C1-C3, 2010.

7. Zovkic IB, Guzman-Karlsson MC and Sweatt JD: Epigenetic regulation of memory formation and maintenance. Learn Mem 20: 61-74, 2013

8. Kim S and Kaang BK: Epigenetic regulation and chromatin remodeling in learning and memory. Exp Mol Med 49: e281, 2017.

9. Peleg S, Sananbenesi F, Zovoilis A, Burkhardt S, Bahari-Javan S, Agis-Balboa RC, Cota P, Wittnam JL, Gogol-Doering A, Opitz $\mathrm{L}$, et al: Altered histone acetylation is associated with age-dependent memory impairment in mice. Science 328: 753-756, 2010.

10. Peixoto $\mathrm{L}$ and Abel $\mathrm{T}$ : The role of histone acetylation in memory formation and cognitive impairments. Neuropsychopharmacology 38 62-76, 2013.

11. MacDonald JL and Roskams AJ: Epigenetic regulation of nervous system development by DNA methylation and histone deacetylation. Prog Neurobiol 88: 170-183, 2009.

12. Sun J, Sun J, Ming GL and Song H: Epigenetic regulation of neurogenesis in the adult mammalian brain. Eur J Neurosci 33: 1087-1093, 2011

13. Hsieh $\mathrm{J}$ and Eisch AJ: Epigenetics, hippocampal neurogenesis, and neuropsychiatric disorders: Unraveling the genome to understand the mind. Neurobiol Dis 39: 73-84, 2010.

14. Simonini MV, Camargo LM, Dong E, Maloku E, Veldic M, Costa $\mathrm{E}$ and Guidotti A: The benzamide MS-275 is a potent, long-lasting brain region-selective inhibitor of histone deacetylases. Proc Natl Acad Sci USA 103: 1587-1592, 2006.

15. Mousa A and Bakhiet M: Role of cytokine signaling during nervous system development. Int J Mol Sci 14: 13931-13957, 2013

16. Thomas GM and Huganir RL: MAPK cascade signalling and synaptic plasticity. Nat Rev Neurosci 5: 173-183, 2004.

17. Peng S, Zhang Y, Zhang J, Wang $\mathrm{H}$ and Ren B: ERK in learning and memory: A review of recent research. Int J Mol Sci 11: 222-232, 2010

18. Sharma SK and Carew TJ: The roles of MAPK cascades in synaptic plasticity and memory in Aplysia: Facilitatory effects and inhibitory constraints. Learn Mem 11: 373-378, 2004.

19. Wang WY, Yang R, Hu SF, Wang H, Ma ZW and Lu Y: $\mathrm{N}$-stearoyl-L-tyrosine ameliorates sevoflurane induced neuroapoptosis via MEK/ERK1/2 MAPK signaling pathway in the developing brain. Neurosci Lett 541: 167-172, 2013.

20. Bi C, Cai Q, Shan Y, Yang F, Sun S, Wu X and Liu H: Sevoflurane induces neurotoxicity in the developing rat hippocampus by upregulating connexin 43 via the JNK/c-Jun/AP-1 pathway. Biomed Pharmacother 108: 1469-1476, 2018.

21. Ji M, Dong L, Jia M, Liu W, Zhang M, Ju L, Yang J, Xie Z and Yang $\mathrm{J}$ : Epigenetic enhancement of brain-derived neurotrophic factor signaling pathway improves cognitive impairments induced by isoflurane exposure in aged rats. Mol Neurobiol 50: 937-944, 2014.

22. AVMA Guidelines for the Euthanasia of Animals: Edition. American Veterinary Medical Association, 2013. https://www.avma.org/resources-tools/avma-policies/avmaguidelines-euthanasia-animals.
23. Livak KJ and Schmittgen TD: Analysis of relative gene expression data using real-time quantitative PCR and the $2(-\Delta \Delta$ C(T)) Method. Methods 25: 402-408, 2001.

24. Vorhees CV and Williams MT: Assessing spatial learning and memory in rodents. ILAR J 55: 310-332, 2014.

25. Chakrabarti A, Oehme I, Witt O, Oliveira G, Sippl W, Romier C, Pierce RJ and Jung M: HDAC8: A multifaceted target for therapeutic interventions. Trends Pharmacol Sci 36: 481-492, 2015.

26. Guo X, Ruan H, Li X, Qin L, Tao Y, Qi X, Gao J, Gan L, Duan S and Shen W: Subcellular Localization of Class I Histone Deacetylases in the Developing Xenopus tectum. Front Cell Neurosci 9: 510, 2016

27. Ma K, Qin L, Matas E, Duffney LJ, Liu A and Yan Z: Histone deacetylase inhibitor MS-275 restores social and synaptic function in a Shank3-deficient mouse model of autism. Neuropsychopharmacology 43: 1779-1788, 2018.

28. Sanders RD, Xu J, Shu Y, Januszewski A, Halder S, Fidalgo A, Sun P, Hossain M, Ma D and Maze M: Dexmedetomidine attenuates isoflurane-induced neurocognitive impairment in neonatal rats. Anesthesiology 110: 1077-1085, 2009.

29. Covington HE III, Maze I, LaPlant QC, Vialou VF, Ohnishi YN, Berton O, Fass DM, Renthal W, Rush AJ III, Wu EY, et al: Antidepressant actions of histone deacetylase inhibitors. J Neurosci 29: 11451-11460, 2009.

30. Choo QY, Ho PC, Tanaka Y and Lin HS: The histone deacetylase inhibitors MS-275 and SAHA suppress the p38 mitogen-activated protein kinase signaling pathway and chemotaxis in rheumatoid arthritic synovial fibroblastic E11 cells. Molecules 18: 14085-14095, 2013.

31. Ferguson BS, Harrison BC, Jeong MY, Reid BG, Wempe MF, Wagner FF, Holson EB and McKinsey TA: Signal-dependent repression of DUSP5 by class I HDACs controls nuclear ERK activity and cardiomyocyte hypertrophy. Proc Natl Acad Sci USA 110: 9806-9811, 2013.

32. Guan QH, Pei DS, Zhang QG, Hao ZB, Xu TL and Zhang GY: The neuroprotective action of SP600125, a new inhibitor of JNK, on transient brain ischemia/reperfusion-induced neuronal death in rat hippocampal CA1 via nuclear and non-nuclear pathways. Brain Res 1035: 51-59, 2005.

33. Kapfhamer D, King I, Zou ME, Lim JP, Heberlein U and Wolf FW: JNK pathway activation is controlled by Tao/TAOK3 to modulate ethanol sensitivity. PLoS One 7: e50594, 2012.

34. Li Y, Wang F, Liu C, Zeng M, Han X, Luo T, Jiang W, Xu J and Wang H: JNK pathway may be involved in isoflurane-induced apoptosis in the hippocampi of neonatal rats. Neurosci Lett 545: $17-22,2013$

This work is licensed under a Creative Commons Attribution-NonCommercial-NoDerivatives 4.0 International (CC BY-NC-ND 4.0) License. 